\title{
Systematic review and meta-analysis appraising efficacy and safety of adrenaline for adult cardiopulmonary resuscitation
}

\author{
Kobi Ludwin ${ }^{1}$, Kamil Safiejko ${ }^{2}$, Jacek Smereka ${ }^{1,3}$, Klaudiusz Nadolny ${ }^{4,5}$,

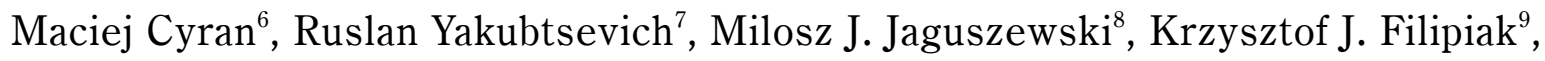 \\ Lukasz Szarpak ${ }^{1,2,6}$, Antonio Rodríguez-Núñez ${ }^{10}$ \\ ${ }^{1}$ Polish Society of Disaster Medicine, Warsaw, Poland \\ ${ }^{2}$ Bialystok Oncology Center, Bialystok, Poland \\ ${ }^{3}$ Department of Emergency Medical Service, Wroclaw Medical University, Wroclaw, Poland \\ ${ }^{4}$ Department of Emergency Medical Service, Higher School of Strategic Planning in Dabrowa Gornicza, Poland \\ ${ }^{5}$ Faculty of Medicine, Katowice School of Technology, Katowice, Poland \\ ${ }^{6}$ Maria Sklodowska-Curie Medical Academy in Warsaw, Poland \\ ${ }^{7}$ Department of Anesthesiology and Intensive Care, Grodno State Medical University, Grodno, Belarus \\ ${ }^{8}$ First Department of Cardiology, Medical University of Gdansk, Poland \\ ${ }^{9}$ First Chair and Department of Cardiology, Medical University of Warsaw, Poland \\ ${ }^{10}$ Pediatric Intensive Care Unit, University Hospital of Santiago de Compostela, Spain
}

\section{This paper was guest edited by Prof. Togay Evrin}

\begin{abstract}
Background: There is a beneficial effect of adrenaline during adult cardiopulmonary resuscitation (CPR) from cardiac arrest but there is also uncertainty about its safety and effectiveness. The aim of this study was to evaluate the use of adrenaline versus non-adrenaline CPR.

Methods: PubMed, ScienceDirect, Embase, CENTRAL (Cochrane Central Register of Controlled Trials) and Google Scholar databases were searched from their inception up to $1^{\text {st }}$ July 2020. Two reviewers independently assessed eligibility and risk of bias, with conflicts resolved by a third reviewer. Risk ratio $(R R)$ or mean difference of groups were calculated using fixed or random-effect models.

Results: Nineteen trials were identified. The use of adrenaline during CPR was associated with a significantly higher percentage of return of spontaneous circulation (ROSC) compared to non-adrenaline treatment (20.9\% vs. 5.9\%; $R R=1.87 ; 95 \%$ confidence interval [CI] 1.37-2.55; $p<0.001$ ). The use of adrenaline in CPR was associated with ROSC at $19.4 \%$ and for non-adrenaline treatment - $4.3 \%$ $(R R=3.23 ; 95 \%$ CI 1.89-5.53; $p<0.001)$. Survival to discharge (or 30-day survival) when using adrenaline was $6.8 \%$ compared to non-adrenaline treatment (5.5\%; $R R=0.99 ; 95 \%$ CI 0.76-1.30; $p=0.97)$. However, the use of adrenaline was associated with a worse neurological outcome $(1.6 \% \mathrm{vs}$. $2.2 \% ; R R=0.57 ; 95 \%$ CI 0.42-0.78; $p<0.001$ ).

Conclusions: This review suggests that resuscitation with adrenaline is associated with the ROSC and survival to hospital discharge, but no higher effectiveness was observed at discharge with favorable neurological outcome. The analysis showed higher effectiveness of ROSC and survival to hospital discharge in non-shockable rhythms. But more multicenter randomized controlled trials are needed in the future. (Cardiol J 2021; 28, 2: 279-292)
\end{abstract}

Key words: adrenaline, epinephrine, cardiac arrest, cardiopulmonary resuscitation, outcome, return of spontaneous circulation, meta-analysis, systematic review

Address for correspondence: Lukasz Szarpak, Assoc. Prof. PhD, MBA, Bialystok Oncology Center, ul. Ogrodowa 12, 15-027 Białystok, Poland, tel: +48 500186225, e-mail: lukasz.szarpak@gmail.com

Received: 12.08 .2020

Accepted: 3.09.2020

This article is available in open access under Creative Common Attribution-Non-Commercial-No Derivatives 4.0 International (CC BY-NC-ND 4.0) license, allowing to download articles and share them with others as long as they credit the authors and the publisher, but without permission to change them in any way or use them commercially. 


\section{Introduction}

Sudden cardiac arrest (SCA) is a challenge for medical personnel, especially in the context of emergency medical teams, where there are a limited number of personnel in the resuscitation team $[1,2]$. Adrenaline has been a key component of advanced life support algorithms for many years. Adrenaline is a catecholamine, showing sympathomimetic activity dependent on direct or indirect stimulation of $\alpha 1, \alpha 2, \beta 1, \beta 2$ receptors. For cardiopulmonary resuscitation (CPR), the effect on $\alpha 1$ receptors is significant due to vasoconstriction. This increases the aortic diastolic pressure, which increases coronary perfusion pressure and cerebral perfusion pressure. As numerous studies indicate, coronary perfusion pressure is closely correlated with the survival of cardiac arrest [3, 4]. It is recommended by both the European Resuscitation Council (ERC) [5], as well as the American Heart Association (AHA) [6]. The use of adrenaline during CPR does not have the highest class of recommendations. Although adrenaline can improve global cerebral and coronary blood flow, due to its vascular contraction, the microcirculatory flow may be reduced $[7,8]$. There is a consistent pattern in studies that suggests that adrenaline can initially resume heart function and increase chances of survival, but can generally increase brain injury [9].

The objective herein, was to compare the survival to hospital discharge rates in patients with cardiac arrest treated with and without adrenaline. In this meta-analysis, we hypothesized that adrenaline confirms benefit over placebo or non-adrenaline treatment under adult CPR as seen by the rate of return of spontaneous circulation (ROSC) and survival to hospital discharge.

\section{Methods}

This systematic review and meta-analysis were conducted following the Preferred Reporting Items for Systematic Reviews and Meta-Analyses (PRISMA) statement for conducting and reporting results [10] and The Meta-analysis Of Observational Studies in Epidemiology (MOOSE) guidelines [11] for observational studies. The systematic review protocol has not been registered. Ethical approval was not required for this meta-analysis.

\section{Literature search strategy and inclusion criteria}

An electronic database search without language restrictions was performed in a standardized, unblinded manner by two independent reviewers (K.L. and M.C.). Inter-reviewer disagreements were resolved by consultation of the third author (J.S.). The search strategy was first applied to PubMed, Web of Science, Embase, ScienceDirect, the Cochrane Central Register of Controlled Trials (CENTRAL) databases from their inception, to July 1,2020. In addition to these sources, manual searches in Google and Google Scholar, and web pages of reliable organizations (gray literature) were conducted. An additional manual cross-reference and related-article search was conducted to identify articles that were not found through prior searches.

Inclusive criteria: (a) Research types: randomized controlled trials, quasi-randomized trials, observational studies; (b) Research subjects: human studies involved adult patients with cardiac arrest were included in our meta-analysis. Studies which were preprint were also included. Case-control studies, non-trials conducted on simulated models, editorials, reviews, guidelines, meta-analysis and theoretical models were excluded from the review.

The following search terms were used: "adrenaline" OR "epinephrine" AND "cardiac arrest" OR "heart arrest" OR "circulation arrest" OR "circulatory arrest" OR "induced heart arrest" OR "heart stand still" OR "cardiac ventric* fibrillation" OR "heart ventric* fibrillation" OR "pulseless ventric* tachycardia" OR “asysto*” OR "pulseless electrical activity”.

\section{Data extraction}

Two independent reviewers conducted the data extraction and checked by each other (K.L. and J.S.). A third reviewer (L.S.) was available to resolve cases for which eligibility was unclear. For each study, a record of the first author, publication time, sample size, country, research type, the primary and secondary measures; inclusion and exclusion criteria; and study quality was included.

\section{Outcomes}

The primary outcome of the current meta-analysis was survival to discharge, defined as the rate of survival to hospital discharge or survival at 30 days. The secondary outcome was the ROSC and survival to discharge with favorable neurological outcome defined as a score of 3 or less on the modified Rankin scale [12] or 14 or 15 points in Glasgow Coma Scale [13].

\section{Quality assessment of included studies \\ Quality assessment was performed by two reviewers (K.S. and K.J.F.). Inter-reviewer disa-}


greements were resolved by consultation (J.S.). For quality assessment of randomized controlled trials (RCTs), the Cochrane Collaboration risk assessment tool for RCTs was used. Studies were graded as "low risk", "high risk" or "unclear" for: random sequence generation, allocation concealment, blinding of participants and personnel, blinding of outcome assessment, incomplete outcome data, selective reporting, and other biases. The review authors' judgments about each risk of bias item are provided in the Supplementary Digital File 1. The Newcastle-Ottawa quality assessment scale was used to appraise the outcome of interest for the cohort study. The modified Newcastle-Ottawa scale was used for the cross-sectional study [14] and is shown in Supplementary Digital File 1.

\section{Statistical analysis}

All statistical analyses were performed with Review Manager Software 5.4 (The Cochrane Collaboration, Oxford, Copenhagen, Denmark) to carry out the single-arm meta-analysis. Outcomes were summarized using the Mantel-Haenszel risk ratios (RRs) or mean differences with a $95 \%$ confidence interval (CI). When the continuous outcome was reported in a study as median, range, and interquartile range, means and standard deviations were estimated using the formula described by Hozo et al. [15]. Heterogeneity was quantitatively evaluated by $\mathrm{I}^{2}$ statistic (no heterogeneity, $\mathrm{I}^{2}=0-25 \%$; moderate heterogeneity, $\mathrm{I}^{2}=25-50 \%$; large heterogeneity, $\mathrm{I}^{2}=50-75 \%$; extreme heterogeneity, $\left.\mathrm{I}^{2}=75-100 \%\right)$. The random-effects model was used for $\mathrm{I}^{2}>50 \%$; otherwise, the fixed effects model was employed. All statistical tests were two-sided and were considered when $\mathrm{p}<0.05$.

\section{Results}

The systematic literature search identified 1282 relevant publications. After the review of titles and abstracts, 45 studies were selected as being potentially eligible for inclusion into this systematic review. After reading the full-text articles, 5 RCTs (published between 1995 and 2018) including 4951 participants [16-20] and 14 nonrandomized trials (published between 1994 and 2016) including 91,537 participants [13, 21-33] were finally included (Fig. 1). Other information was listed in the Tables 1 and 2 of characteristics of included studies.

\section{Return of spontaneous circulation}

Twelve studies reported ROSC [13, 16-18, 20-22, 24-28]. Polled analysis showed that the use of adrenaline during CPR was associated with a significantly higher percentage of ROSC compared to non-adrenaline treatment $(20.9 \%$ vs. $5.9 \% ; \mathrm{RR}=1.87 ; 95 \%$ CI $1.37-2.55 ; \mathrm{p}<0.001$; Fig. 2). The above trend was reflected in both RCTs (35.9\% vs. $12.8 \%$; $\mathrm{RR}=2.28$; $95 \%$ CI $1.49-3.49$; $\mathrm{p}<0.001)$ and observational studies $(19.9 \%$ vs. $5.8 \%$; RR $=1.70 ; 95 \%$ CI 1.15-2.53; $\mathrm{p}=0.009$ ).

The incidence of ROSC for shockable rhythms for adrenaline use was $24.0 \%$ and $28.1 \%$ for non-adrenaline use $(\mathrm{RR}=0.86 ; 95 \%$ CI $0.77-0.96$; $\mathrm{p}=0.007)$. For non-shockable rhythms, the reverse trend was observed (Fig. 3). The use of adrenaline in the CPR process was associated with ROSC at $19.4 \%$ and for non-adrenaline treatment $-4.3 \%(\mathrm{RR}=3.23$; 95\% CI 1.89-5.53; $\mathrm{p}<0.001)$.

\section{Survival to discharge}

Survival to discharge (or 30 day survival) using adrenaline was $6.8 \%$ compared to the non-adrenaline treatment $(5.5 \%$; $\mathrm{RR}=0.99 ; 95 \% \mathrm{CI}$ 0.76-1.30; $p=0.97$; Fig. 4) [16-18, 20-24, 26-33].

In the case of non-shockable rhythms, the use of adrenaline compared to non-adrenaline treatment was associated with higher survival to hospital discharge rate (3.9\% vs. $2.9 \%$, respectively; $\mathrm{RR}=1.16$; 95\% CI 0.86-1.55; $\mathrm{p}=0.32$; Fig. 5) [17, 21, 22, 24, 28-30, 33]. For shockable rhythms, higher survival to discharge was observed in the non-adrenaline group compared to the adrenaline group $(27.1 \%$ vs. $15.7 \%$, respectively; $\mathrm{RR}=0.63$; 95\% CI 0.56-0.70; p < 0.001) [17, 21, 22, 28, 29, 33].

\section{Survival to discharge with favorable neurological outcome}

Ten studies [13, 16, 17, 20-22, 24, 26-28] reported survival to discharge with a favorable neurological outcome and indicated that the use of adrenaline was associated with worse outcome (1.6\% vs. $2.2 \%$; $\mathrm{RR}=0.57 ; 95 \%$ CI 0.42-0.78; $\mathrm{p}<0.001)$.

In randomized clinical trials $[16,17,20]$, the use of adrenaline was associated with a slightly higher percentage of patients with survival and favorable neurological outcome compared to the non-adrenaline group (2.9\% vs. $2.4 \%$; $\mathrm{RR}=1.21$; 95\% CI 0.95-1.54; $\mathrm{p}=0.13$ ). The opposite trend was observed for observational studies (Suppl. Digital File 1) [13, 21, 22, 24, 26-28].

The analysis in subgroups concerning the type of rhythm showed that in cases of shockable 


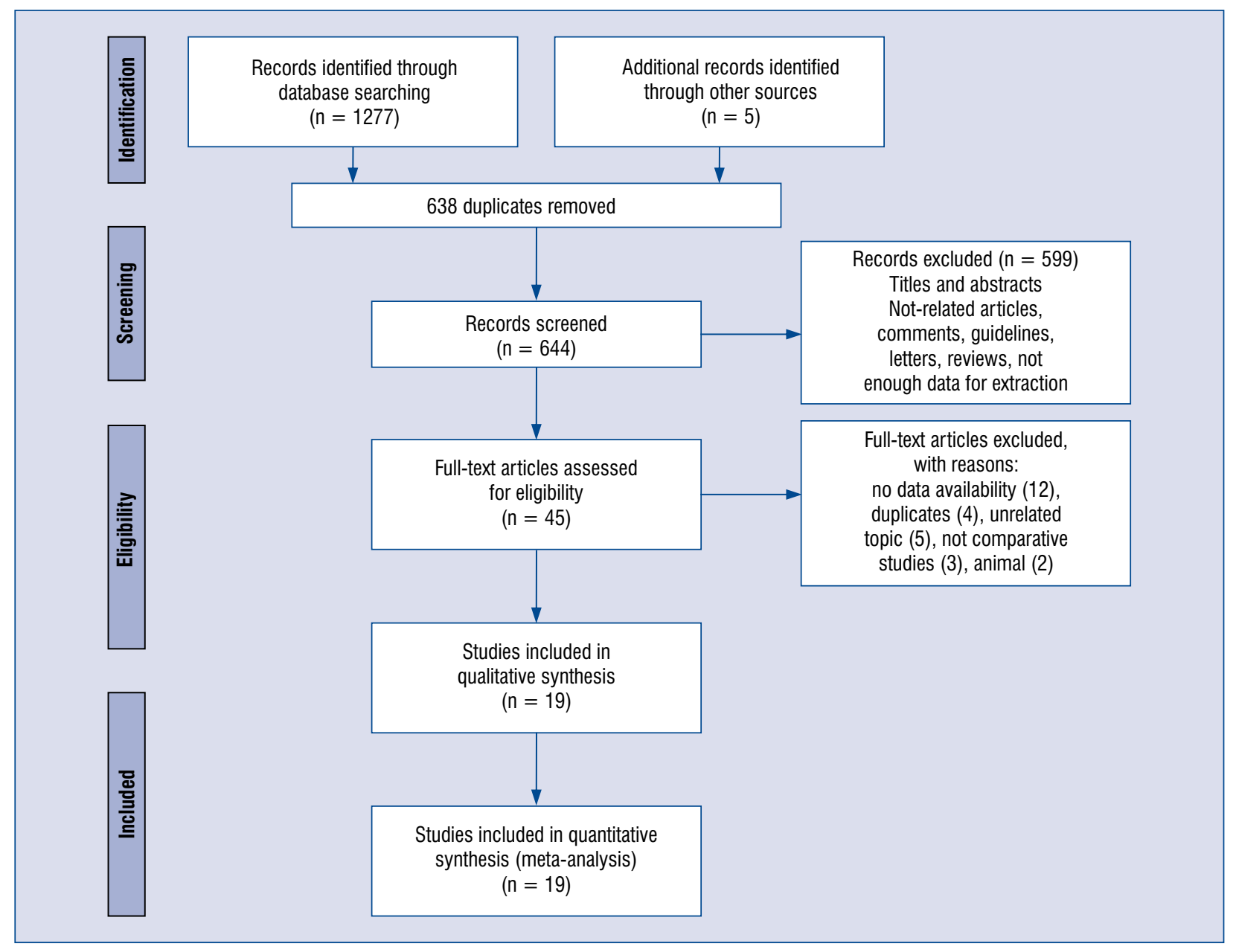

Figure 1. Flow diagram showing stages of database searching and study selection.

rhythms, the use of adrenaline was associated with statistically significant worse prognosis (survival to discharge with the favorable neurological outcome) than the non-adrenaline group $(7.4 \%$ vs. $19.1 \%$, respectively; $\mathrm{RR}=0.40 ; 95 \% \mathrm{CI} 0.35-0.45 ; \mathrm{p}<$ $<0.001$; Suppl. Digital File 1) [21, 22, 24, 28, 33]. For non-adrenaline rhythms the outcome was comparable and was $0.8 \%$ vs. $0.9 \%$, respectively $(R R=$ $=0.94 ; 95 \%$ CI $0.16-5.50 ; \mathrm{p}=0.94)[21,22,28,33]$.

\section{Long-period outcome}

Two studies reported 3-month survival rates $[16,20]$. Higher survival rates were observed for adrenaline $(3.7 \%)$, while for non-adrenaline treatment the survival rate was $2.8 \%(\mathrm{RR}=1.34$; 95\% CI 1.06-1.68; $\mathrm{p}=0.01)$. One study, Perkins et al. [16] reported good neurological outcome at 3 months. Better results were obtained with adrenaline compared to the non-adrenaline group $(2.1 \%$ vs. $1.6 \% ; \mathrm{RR}=1.30 ; 95 \%$ CI $0.94-1.80 ; \mathrm{p}=0.11)$.

\section{Quality of evidence}

The risk of bias in the included RCTs as well as nonrandomized studies is summarized in Supplementary Digital File 1. Only four studies were randomized controlled trials. The risk of bias was assessed as low or moderate in most of the studies.

\section{Discussion}

The main finding was as follows: (1) the use of adrenaline increased the chances of ROSC; (2) adrenaline was associated with increased survival to hospital discharge rate, however, survival to discharge with favorable neurological outcome was better in the non-adrenaline group.

Studies published in recent years on the use of adrenaline in SCA are extremely important because of the large number of participants and also because of their randomized nature with 


\begin{tabular}{|c|c|c|c|c|c|c|c|c|c|c|c|c|c|c|}
\hline$\Phi$ & 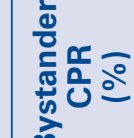 & 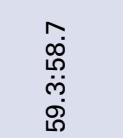 & 兽 & 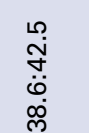 & $\frac{\pi}{z}$ & 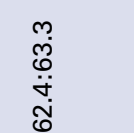 & 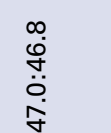 & 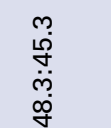 & $\begin{array}{l}\infty \\
0 \\
0 \\
0 \\
0 \\
0.0\end{array}$ & 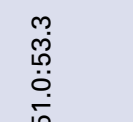 & 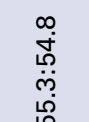 & $\frac{\pi}{z}$ & $\frac{\pi}{z}$ & 芦 \\
\hline 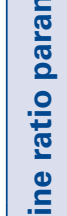 & 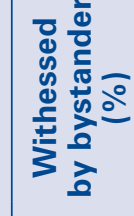 & 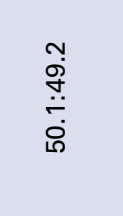 & 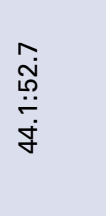 & 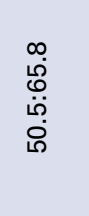 & $\frac{\mathbb{1}}{2}$ & $\begin{array}{l}\stackrel{0}{0} \\
\stackrel{0}{0} \\
\hat{0}\end{array}$ & $\begin{array}{l}\overline{0} \\
0 \\
0 \\
0 \\
0 \\
0\end{array}$ & 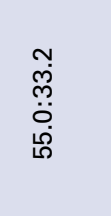 & 弪 & 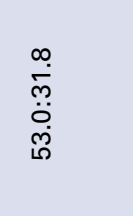 & 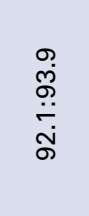 & $\frac{\pi}{z}$ & 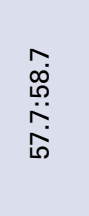 & $\begin{array}{l}\stackrel{\circ}{0} \\
\stackrel{0}{0}\end{array}$ \\
\hline 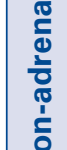 & 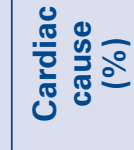 & 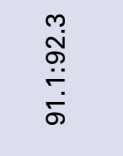 & 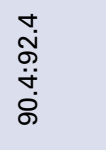 & 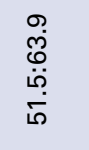 & $\frac{\pi}{2}$ & 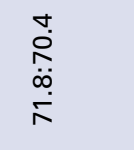 & 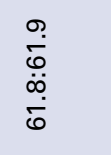 & $\frac{\pi}{z}$ & 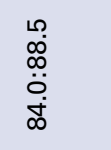 & 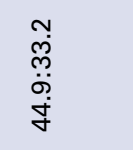 & $\underline{\underline{z}}$ & $\underline{\Sigma}$ & 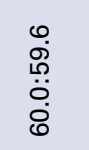 & 密 \\
\hline 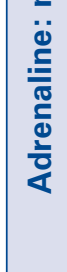 & 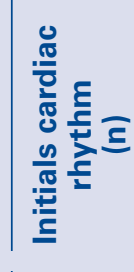 & 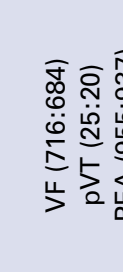 & 5 & 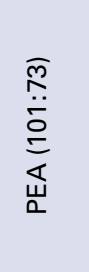 & 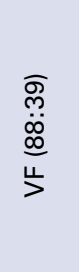 & 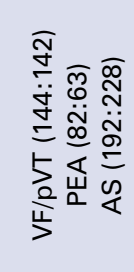 & 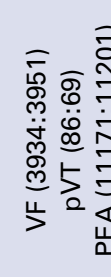 & $\begin{array}{l}3 \\
0 \\
\frac{0}{4} 5\end{array}$ & 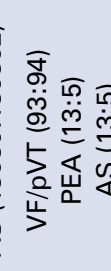 & & 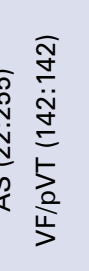 & 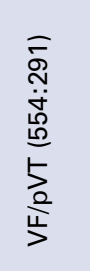 & $\frac{\pi}{z}$ & 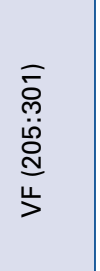 \\
\hline 产 & $\frac{\mathscr{g}}{\tilde{\Phi}}$ & 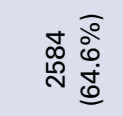 & 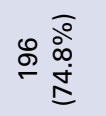 & 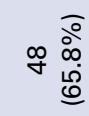 & $\frac{\pi}{2}$ & 哭 & 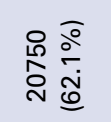 & 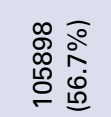 & 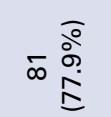 & 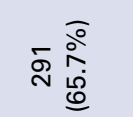 & & $\frac{\pi}{z}$ & 芯总 & 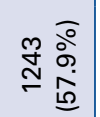 \\
\hline 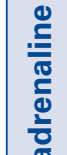 & 这 & 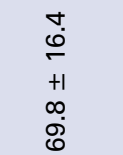 & 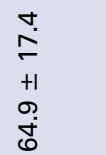 & & 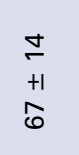 & 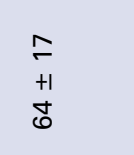 & 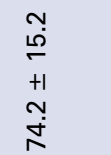 & 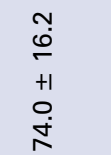 & 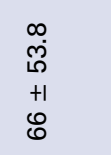 & $\begin{array}{l}\infty \\
\stackrel{\infty}{1} \\
+1 \\
0\end{array}$ & 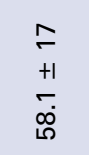 & $\frac{\pi}{2}$ & 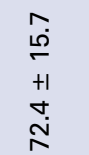 & 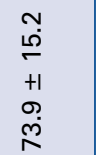 \\
\hline ì & $z$ & 嵒 & $\tilde{N}$ & 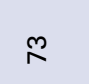 & $\stackrel{\circ}{\circ}$ & 受 & 竔 & 总 & $\stackrel{ \pm}{\stackrel{ \pm}{2}}$ & 孚 & & 产 & 突 & $\stackrel{\frac{\infty}{\sim}}{\sim}$ \\
\hline$\cong$ & $\mid \frac{\mathscr{g}}{\frac{\mathscr{J}}{\tilde{m}}}$ & 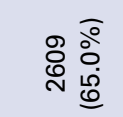 & 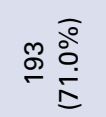 & ஜ & $\frac{\pi}{2}$ & ऽ్ & 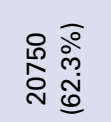 & 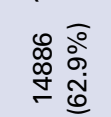 & s. & 空 & & $\frac{s}{z}$ & & : \\
\hline 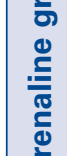 & 兽 & 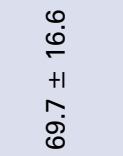 & 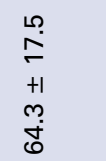 & & 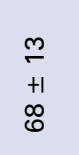 & 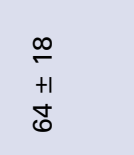 & 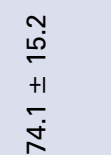 & 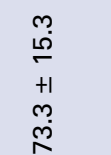 & $\begin{array}{l}\text { o. } \\
0 \\
0 \\
+1 \\
0\end{array}$ & $\begin{array}{l}\infty \\
\stackrel{\infty}{+1} \\
\mathbb{1}\end{array}$ & $\begin{array}{l}0 \\
\stackrel{0}{1} \\
\stackrel{1}{0}\end{array}$ & $\frac{\pi}{z}$ & 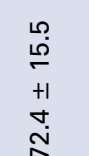 & 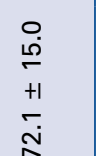 \\
\hline व & $z$ & 号 & $\underset{N}{N}$ & $\stackrel{\bar{\sigma}}{ }$ & 导 & $\stackrel{\infty}{\sigma}$ & 鸹 & : & $\stackrel{\stackrel{9}{=}}{ }$ & g & $\stackrel{\infty}{\sim}$ & $\stackrel{R}{R}$ & 恿 & $\stackrel{m}{D}$ \\
\hline 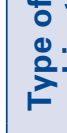 & & $\Sigma$ & $\Sigma$ & 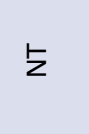 & 5 & $\Sigma$ & $\Sigma$ & $\Sigma$ & 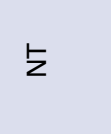 & 5 & 5 & $\Sigma$ & E & $\Sigma$ \\
\hline 黑 & & 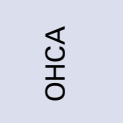 & 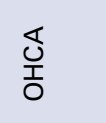 & $\begin{array}{l}\mathbb{\Im} \\
\text { 옹 }\end{array}$ & 总 & $\begin{array}{l}\mathbb{s} \\
\text { 옹 }\end{array}$ & $\begin{array}{l}\widehat{\Phi} \\
\text { I্ }\end{array}$ & $\begin{array}{l}\widehat{\Phi} \\
\text { 옹 }\end{array}$ & $\begin{array}{l}\widehat{\Phi} \\
\text { I্ }\end{array}$ & $\begin{array}{l}\widehat{\Phi} \\
\text { 옹 }\end{array}$ & $\begin{array}{l}\widehat{\Upsilon} \\
\text { 옹 }\end{array}$ & $\begin{array}{l}\widehat{\Phi} \\
\text { 옹 }\end{array}$ & 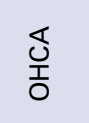 & $\begin{array}{l}\widehat{\Phi} \\
\text { I্ }\end{array}$ \\
\hline 竞 & $\geqq$ & 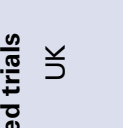 & 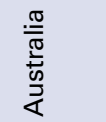 & $\frac{\vec{d}}{\frac{\hat{z}}{2}}$ & 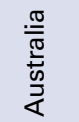 & $\frac{\vec{d}}{\underline{z}}$ & 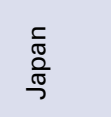 & 产 & $\frac{\bar{d}}{\frac{1}{2}}$ & 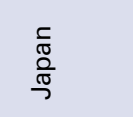 & 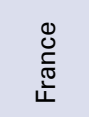 & 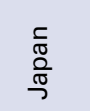 & 高 & 咅 \\
\hline 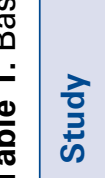 & & 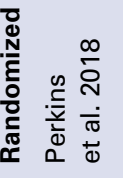 & 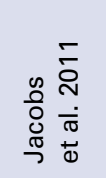 & & & 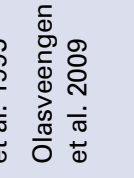 & 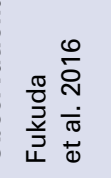 & 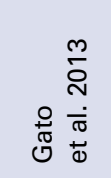 & 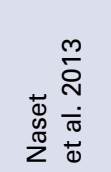 & 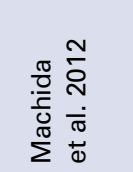 & & & & 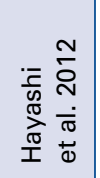 \\
\hline
\end{tabular}




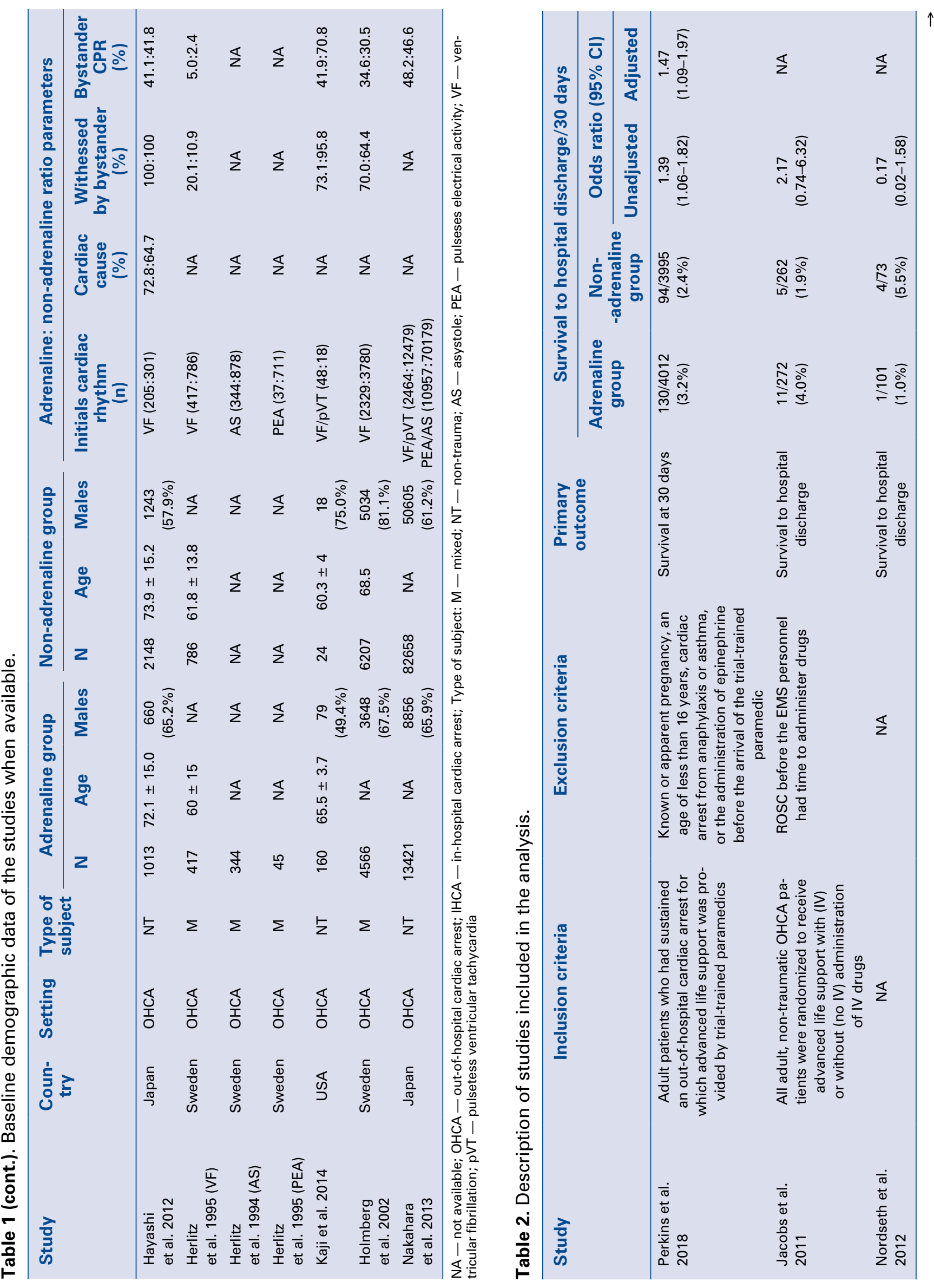




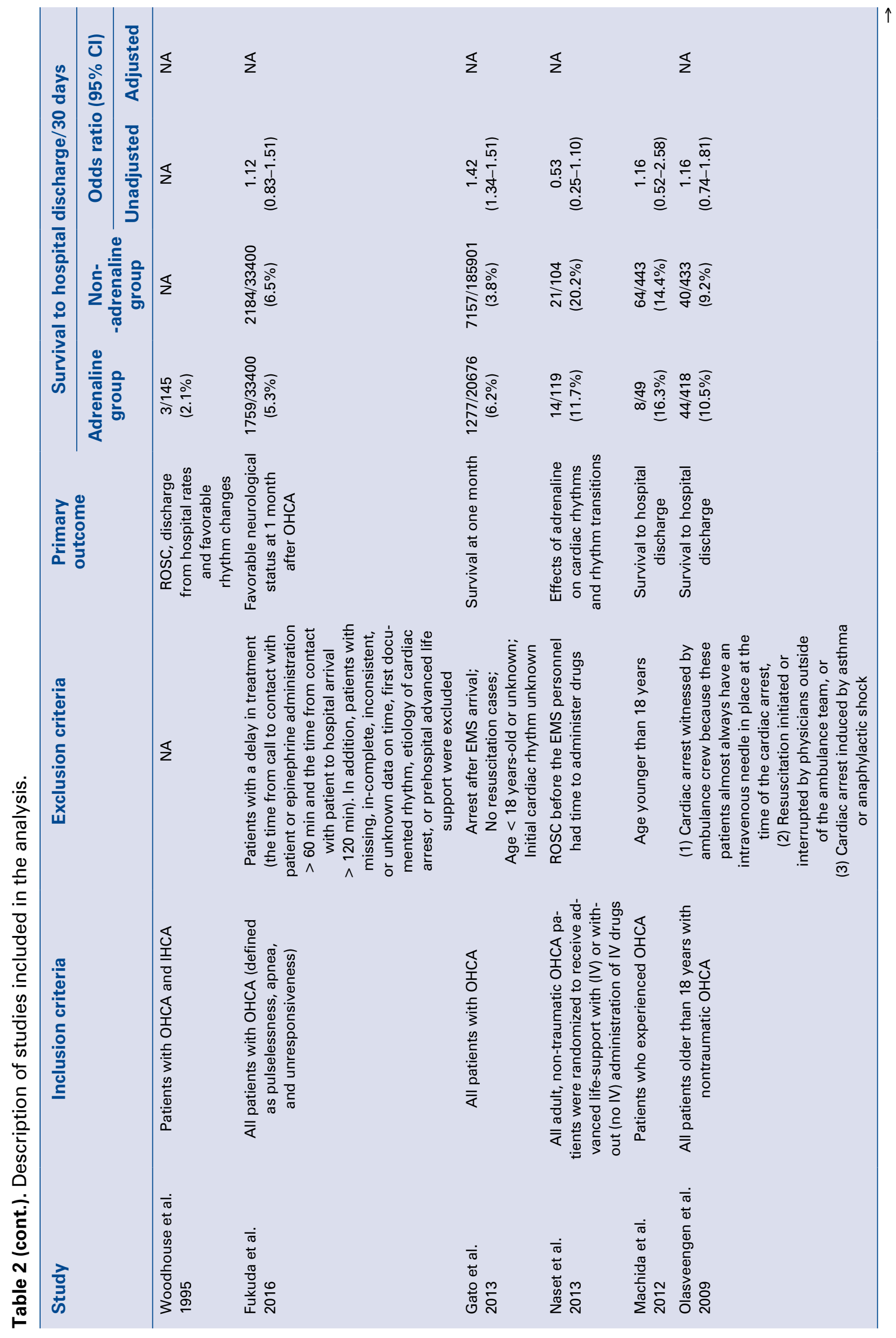




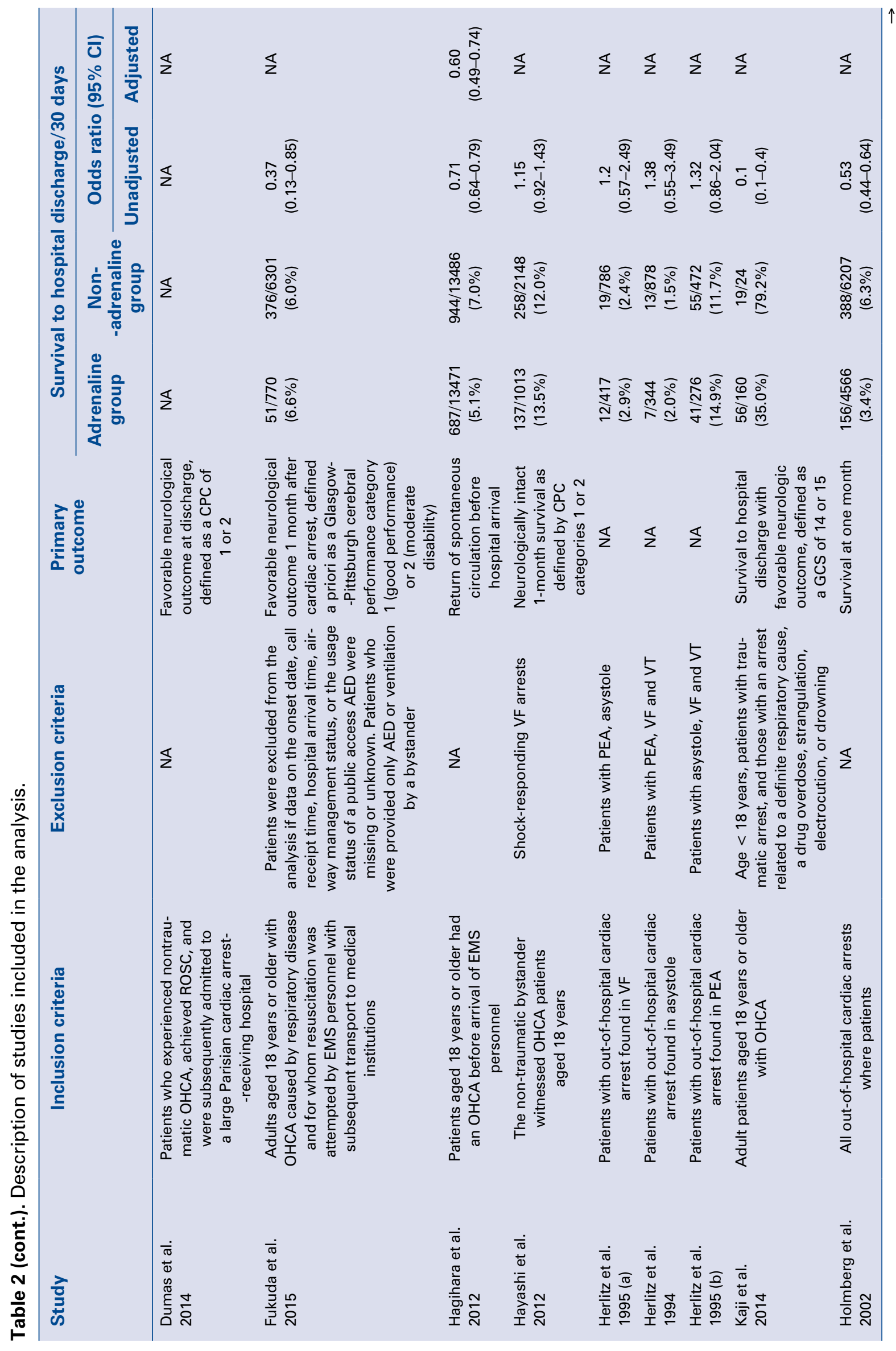




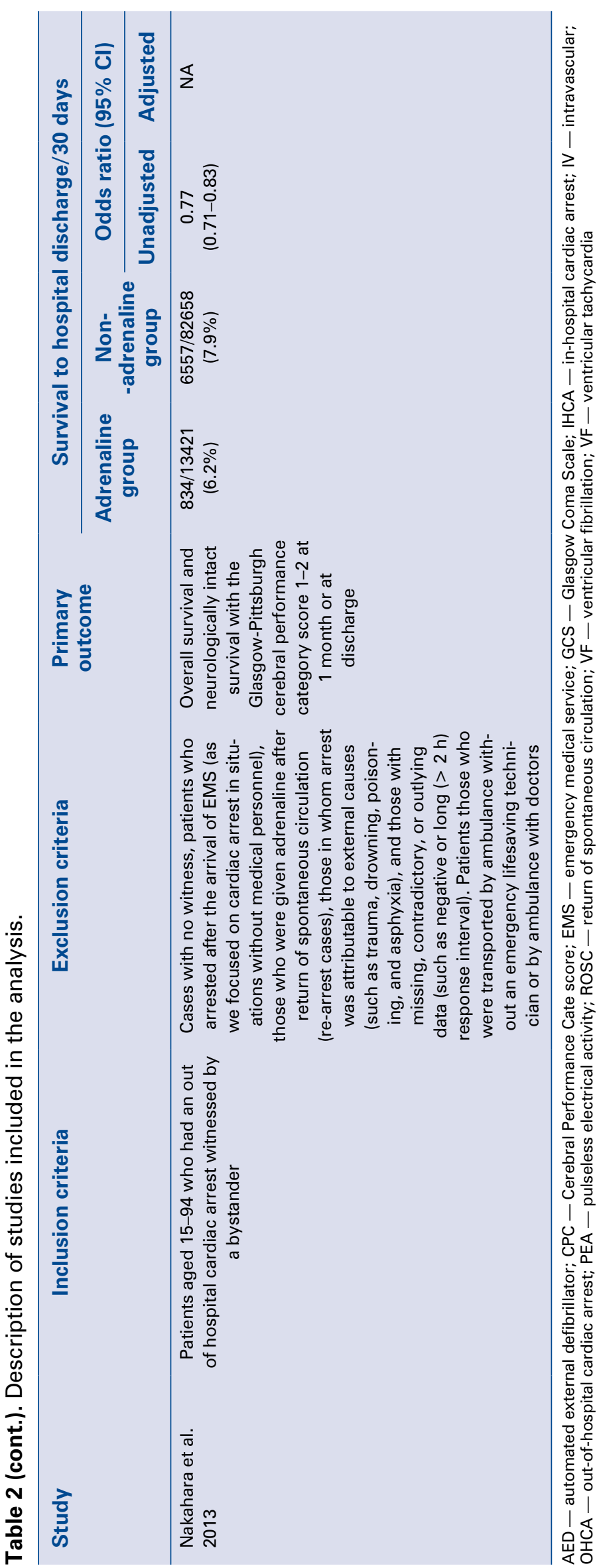

a double-blinded placebo. The data obtained in this meta-analysis again indicates the need to consider the usefulness of routine adrenaline administration in SCA. While the use of adrenaline has been shown to increase the ROSC and survival to hospital discharge, it does not affect the favorable neurological outcome. The results suggest considering routine adrenaline use in case of out-of-hospital SCA.

Return of spontaneous circulation is one of the basic outcomes of resuscitation, especially in the prehospital setting [34]. Pooled analysis showed that the use of adrenaline increases the chance of ROSC, which was evident in both RCTs and observational studies. It was apparent that administration of adrenaline for shockable rhythms was associated with a lower incidence of ROSC. It should be noted, however, that adrenaline is administered according to the guidelines only after ineffective defibrillation, not from the initiation of CPR procedures. In the case of non-shockable rhythms, the difference in ROSC was very significant, ROSC was $19.4 \%$ for adrenaline and $4.3 \%$ for non-adrenaline treatment.

Another important element is survival to discharge, where, as in the case of ROSC, it was observed that for non-shockable rhythms, the use of adrenaline compared to non-adrenaline treatment was associated with higher survival to hospital discharge rate, however, these differences were not statistically significant. Again, as for ROSC for shockable rhythms, higher survival to discharge was observed in the non-adrenaline group.

Survival to discharge with the favorable neurological outcome is essential for the functioning of the patient after the SCA incident with a satisfactory quality of life. In the case of shockable rhythms, the use of adrenaline was associated with a statistically significantly worse prognosis.

The administration of adrenaline in SCA is one of the key elements of resuscitation, especially in cases of non-shockable rhythms [5, 6]. However, it should be noted that there are many milestones in the history of the development of guidelines for resuscitation and many changes have been milestones, including the issue of ratio of chest compressions to the number of breaths, the use of defibrillation, including automated external defibrillator, and improved quality of chest compressions or airway management, where supraglottic airway devices were introduced and less emphasis on the need for endotracheal intubation.

There were also changes in pharmacotherapy in sudden cardiac arrest; over the years, the adrenaline dose was changed, and the rule was introduced 


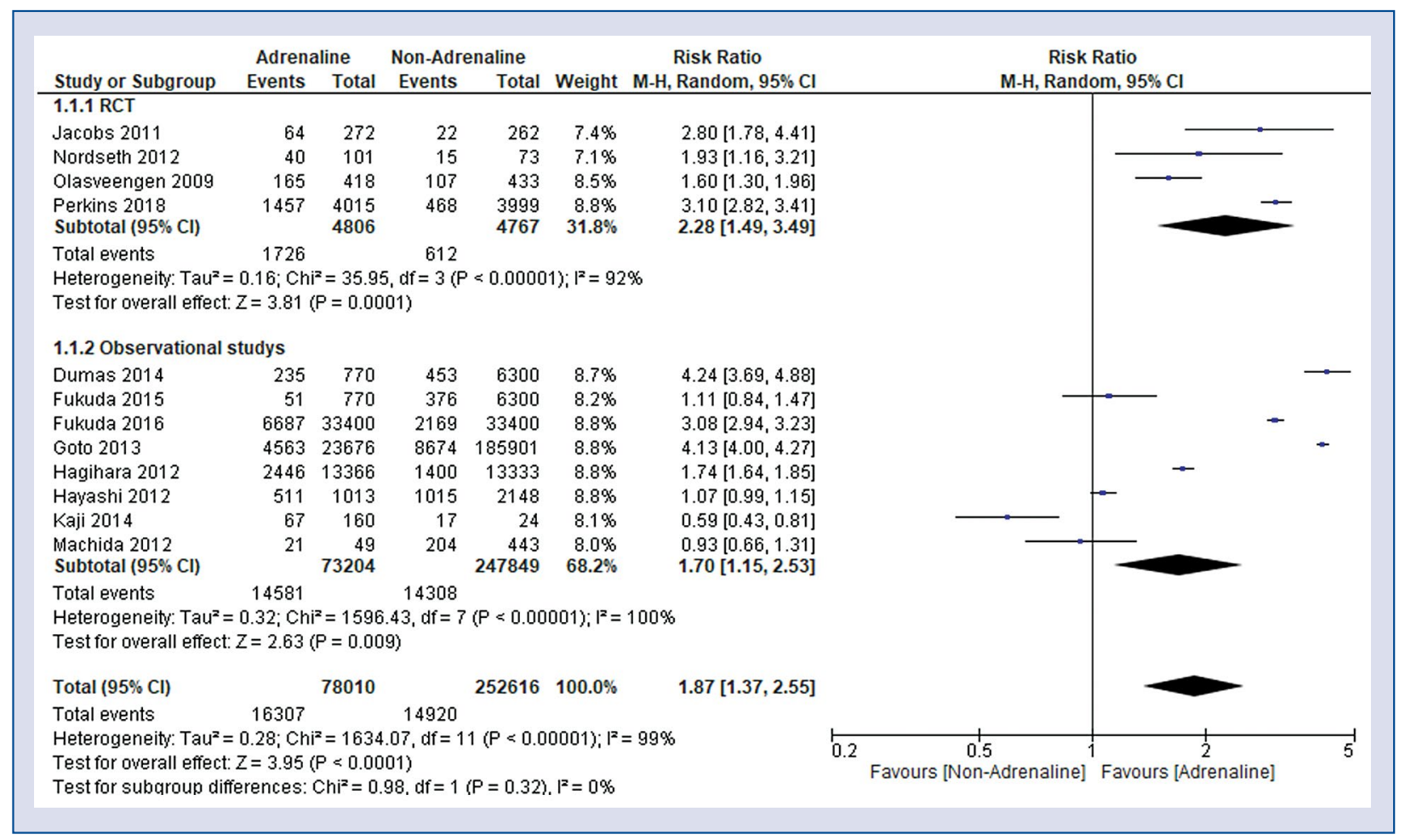

Figure 2. Forest plot of return of spontaneous circulation in adrenaline vs. non-adrenaline groups. The center of each square represents the relative risk for individual trials, and the corresponding horizontal line stands for a $95 \%$ confidence interval (Cl). The diamonds represent pooled results; RCT — randomized controlled trial.

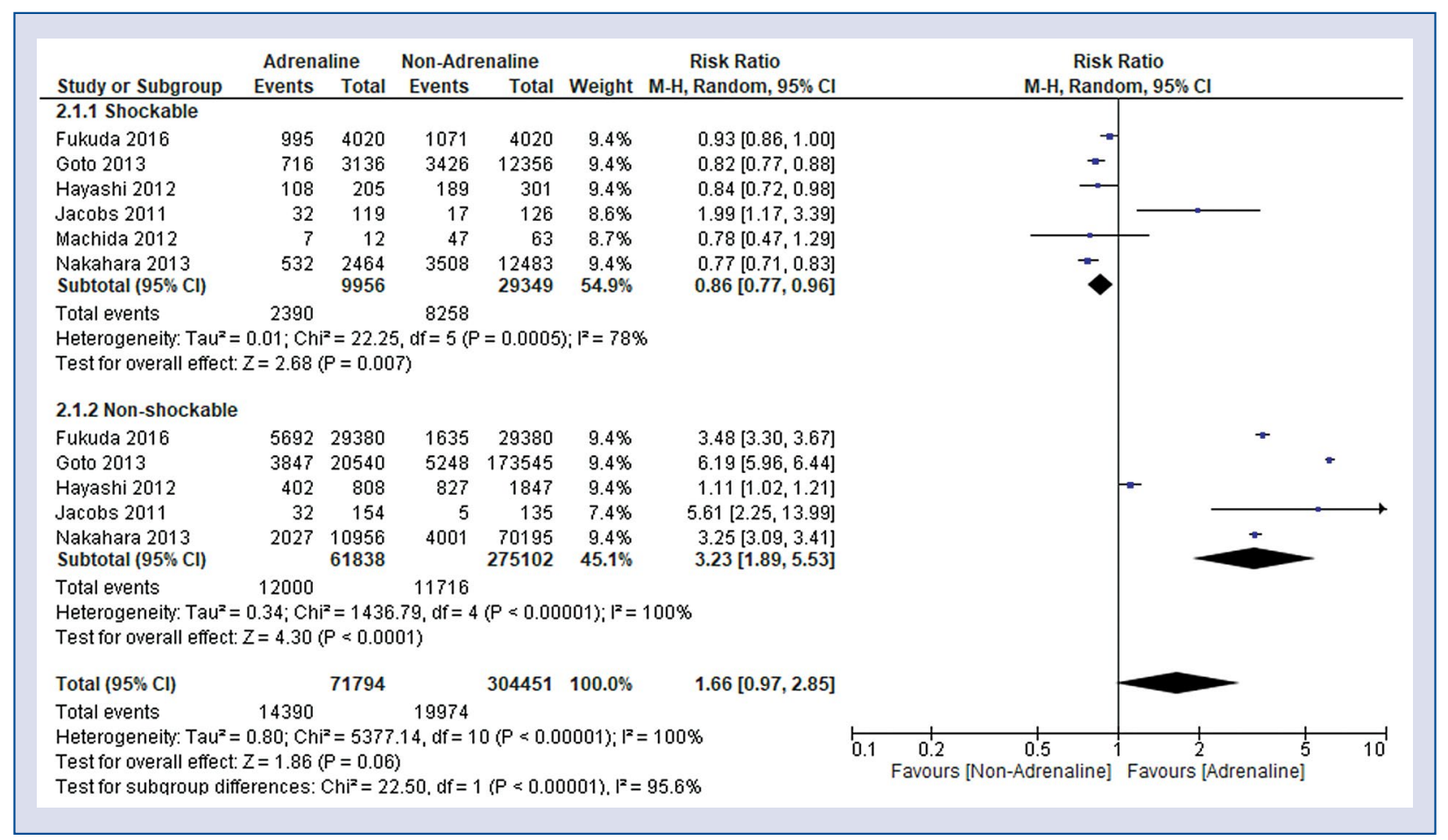

Figure 3. Forest plot of return of spontaneous circulation by type of rhythm in adrenaline vs. non-adrenaline groups. The center of each square represents the relative risk for individual trials, and the corresponding horizontal line stands for a $95 \%$ confidence interval $(\mathrm{Cl})$. The diamonds represent pooled results. 


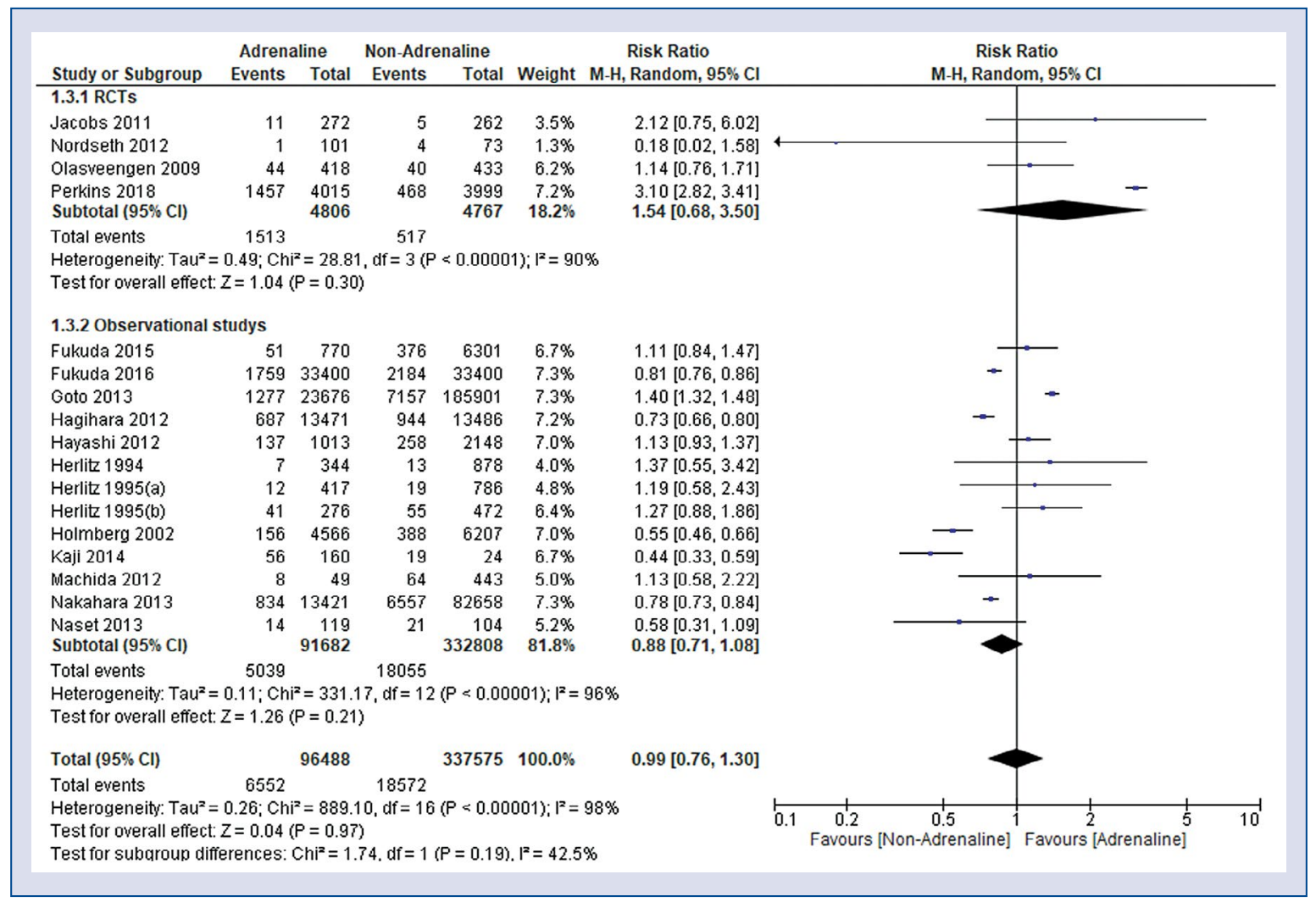

Figure 4. Forest plot of survival to hospital discharge in adrenaline vs. non-adrenaline groups. The center of each square represents the relative risk for individual trials, and the corresponding horizontal line stands for a $95 \%$ confidence interval (CI). The diamonds represent pooled results; RCTs — randomized controlled trials.

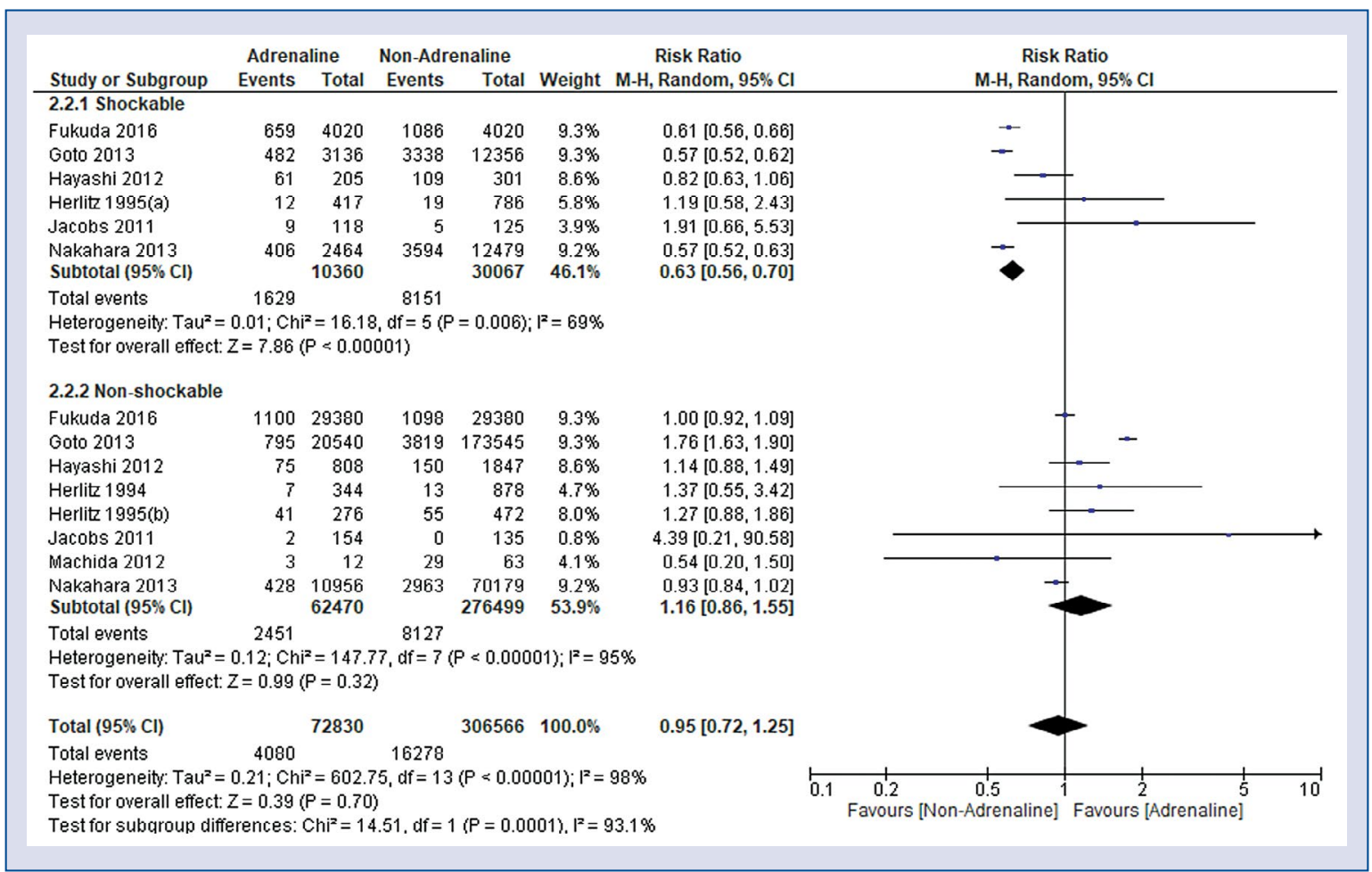

Figure 5. Forest plot of survival to hospital discharge by type of rhythm in adrenaline vs. non-adrenaline groups. The center of each square represents the relative risk for individual trials, and the corresponding horizontal line stands for a $95 \%$ confidence interval (CI). The diamonds represent pooled results. 
that for non-shockable rhythms adrenaline is not administered immediately after SCA recognition. Perhaps the next stage will be the re-analysis of indications for adrenaline administration in SCA at the pre-hospital and hospital stages for shockable and non-shockable rhythms.

Changes in the guidelines and recommendations for resuscitation must be based on further scientific evidence based on high quality randomized clinical trials conducted in both hospital and out-of-hospital settings [35]. Although achieving ROSC is a key task of the resuscitation team, the patient's survival with a favorable neurological outcome is the most important goal and outcome. Both AHA and ERC guidelines are based on the analysis of scientific evidence and the most important are randomized double-blind clinical trials and meta-analyses including pooled data on large patient groups.

The advantage of the meta-analysis is the rigorous application of rules and criteria used in meta-analyses and a thorough search of available databases, as well as references in publications and manual searches in Google and Google Scholar, and the web pages of reliable organizations (gray literature) and analyses of the results obtained as well as following PRISMA statement for conducting and reporting results and The MOOSE guidelines for observational studies.

\section{Limitations of the study}

The results reported in the present systematic review and meta-analysis are subject to several limitations. First, only four studies included in the meta-analysis were randomized controlled trials. Some outcome measures were not uniformly reported across studies and, therefore, were difficult to combine in a meta-analysis. The studies analyzed differed significantly in terms of the number of participants. Another limitation relates to the inclusion of research only in the context of out-of-hospital cardiac arrest. The results of adrenaline administration during CPR in hospital conditions may be different. Therefore, further analyses are planned for in-hospital cardiac arrest. When analyzing the results obtained in this article, all the limitations typical for meta-analyses, including the risk of bias and heterogeneous studies, should also be considered.

Return of spontaneous circulation and the neurological outcome are significantly influenced by the quality of resuscitation, especially the quality of chest compressions [1, 36-38]. Unfortunately, the analyzed studies did not routinely use devices and methods to monitor the quality of chest compression, and chest compression depth and rate, as well as full chest recoil, which has a significant impact on the overall quality of CPR and the overall outcome of the rescue procedure. High-quality chest compressions consist of achieving the correct recommended compression depth, compressions rate, correct chest recoil, minimizing interruptions in chest compressions, as well as the highest possible percentage of correct compressions concerning all compressions carried out with the correct compression site [39-41]. The lack of chest compression quality measurement may affect the results [1], but this effect is reduced by the randomized nature of the double-blinded studies.

The results obtained underline the need for further research on the use of vasopressors in the course of CPR. Another factor to be taken into account is the need to establish a vascular access (intravenous or intraosseous) for the administration of drugs, which may cause difficulties during resuscitation [42]. If the routine supply of adrenaline during $\mathrm{CPR}$ is discontinued, this may result in a lack of immediate need for intravascular access and may further increase the focus on high-quality chest compression, electrotherapy and ventilation and the elimination of potentially reversible causes [5].

\section{Conclusions}

The present meta-analysis demonstrates that resuscitation with adrenaline is associated with the ROSC and survival to hospital discharge, but no higher effectiveness was noted for discharge with favorable neurological outcome. The analysis showed higher effectiveness of ROSC and survival to hospital discharge in non-shockable rhythms. But more multicenter RCTs are needed in the future.

\section{Acknowledgements}

The study was supported by the ERC Research Net and by the Polish Society of Disaster Medicine.

\section{Conflict of interest: None declared}

\section{References}

1. Majer J, Jaguszewski MJ, Frass M, et al. Does the use of cardiopulmonary resuscitation feedback devices improve the quality of chest compressions performed by doctors? A prospective, randomized, cross-over simulation study. Cardiol J. 2019; 26(5): 529_ 535, doi: 10.5603/CJ.a2018.0091, indexed in Pubmed: 30155865. 
2. Malysz M, Kacprzak P. Is low voltage ventricular fibrillation still a diagnostic problem? Disaster Emerg Med J. 2019; 4(1): 31-32, doi: 10.5603/demj.2019.0007.

3. Rojas-Salvador C, Moore JC, Salverda B, et al. Effect of controlled sequential elevation timing of the head and thorax during cardiopulmonary resuscitation on cerebral perfusion pressures in a porcine model of cardiac arrest. Resuscitation. 2020; 149: 162-169, doi: 10.1016/j.resuscitation.2019.12.011, indexed in Pubmed: 31972229.

4. Segal N, Metzger AK, Moore JC, et al. Correlation of end tidal carbon dioxide, amplitude spectrum area, and coronary perfusion pressure in a porcine model of cardiac arrest. Physiol Rep. 2017; 5(17): e13401, doi: 10.14814/phy2.13401, indexed in Pubmed: 28899911.

5. Soar J, Nolan JP, Böttiger BW, et al. European Resuscitation Council Guidelines for Resuscitation 2015: Section 3. Adult advanced life support. Resuscitation. 2015; 95: 100-147, doi: 10.1016/j.resuscitation.2015.07.016, indexed in Pubmed: 26477701.

6. Link MS, Berkow LC, Kudenchuk PJ, et al. Part 7: Adult Advanced Cardiovascular Life Support: 2015 American Heart Association Guidelines Update for Cardiopulmonary Resuscitation and Emergency Cardiovascular Care. Circulation. 2015; 132(18 Suppl 2): S444-S464, doi: 10.1161/CIR.0000000000000261, indexed in Pubmed: 26472995.

7. Krejci V, Hiltebrand LB, Sigurdsson GH. Effects of epinephrine, norepinephrine, and phenylephrine on microcirculatory blood flow in the gastrointestinal tract in sepsis. Crit Care Med. 2006; 34(5): 1456-1463, doi: 10.1097/01.CCM.0000215834.48023.57, indexed in Pubmed: 16557162.

8. Trąbka-Zawicki A, Tomala M, Zeliaś A, et al. Adaptation of global hemostasis to therapeutic hypothermia in patients with out-ofhospital cardiac arrest: Thromboelastography study. Cardiol J. 2019; 26(1): 77-86, doi: 10.5603/CJ.a2017.0080, indexed in Pubmed: 28695976.

9. Ng KT, Teoh WY. The effect of prehospital epinephrine in out-ofhospital cardiac arrest: a systematic review and meta-analysis. Prehosp Disaster Med. 2019; 34(5): 532-539, doi: 10.1017/ S1049023X19004758, indexed in Pubmed: 31455452.

10. Moher D, Shamseer L, Clarke M, et al. PRISMA-P Group. Preferred reporting items for systematic review and meta-analysis protocols (PRISMA-P) 2015 statement. Syst Rev. 2015; 4: 1, doi: 10.1186/2046-4053-4-1, indexed in Pubmed: 25554246.

11. Stroup DF, Berlin JA, Morton SC, et al. Meta-analysis of observational studies in epidemiology: a proposal for reporting. Meta-analysis Of Observational Studies in Epidemiology (MOOSE) group. JAMA. 2000; 283(15): 2008-2012, doi: 10.1001/ jama.283.15.2008, indexed in Pubmed: 10789670.

12. Haywood K, Whitehead L, Nadkarni VM, et al. COSCA (Core Outcome Set for Cardiac Arrest) in Adults: An Advisory Statement From the International Liaison Committee on Resuscitation. Circulation. 2018; 137(22): e783-e801, doi: 10.1161/ CIR.0000000000000562, indexed in Pubmed: 29700122.

13. Kaji AH, Hanif AM, Bosson N, et al. Predictors of neurologic outcome in patients resuscitated from out-of-hospital cardiac arrest using classification and regression tree analysis. Am J Cardiol. 2014; 114(7): 1024-1028, doi: 10.1016/j.amjcard.2014.06.031, indexed in Pubmed: 25118118.

14. Wells GA, Shea B, O'Connell D, et al: The Newcastle-Ottawa Scale (NOS) for assessing the quality of nonrandomised studies in meta-analyses. The Ottawa Hospital Research Institute.
2014. http://www.ohri.ca/programs/clinical_epidemiology/oxford. asp (accessed May 10, 2020).

15. Hozo SP, Djulbegovic B, Hozo I. Estimating the mean and variance from the median, range, and the size of a sample. BMC Med Res Methodol. 2005; 5: 13, doi: 10.1186/1471-2288-5-13, indexed in Pubmed: 15840177.

16. Perkins GD, Ji C, Deakin CD, et al. A randomized trial of epinephrine in out-of-hospital cardiac arrest. N Engl J Med. 2018; 379(8): 711-721, doi: 10.1056/NEJMoa1806842, indexed in Pubmed: 30021076 .

17. Jacobs IG, Finn JC, Jelinek GA, et al. Effect of adrenaline on survival in out-of-hospital cardiac arrest: A randomised double-blind placebo-controlled trial. Resuscitation. 2011; 82(9): 1138-1143, doi: 10.1016/j.resuscitation.2011.06.029, indexed in Pubmed: 21745533.

18. Nordseth T, Olasveengen TM, Kvaløy JT, et al. Dynamic effects of adrenaline (epinephrine) in out-of-hospital cardiac arrest with initial pulseless electrical activity (PEA). Resuscitation. 2012; 83(8): 946-952, doi: 10.1016/j.resuscitation.2012.02.031, indexed in Pubmed: 22429969.

19. Woodhouse SP, Cox S, Boyd P, et al. High dose and standard dose adrenaline do not alter survival, compared with placebo, in cardiac arrest. Resuscitation. 1995; 30(3): 243-249, doi: 10.1016/0300-9572(95)00890-x.

20. Olasveengen TM, Sunde K, Brunborg C, et al. Intravenous drug administration during out-of-hospital cardiac arrest: a randomized trial. JAMA. 2009; 302(20): 2222-2229, doi: 10.1001/ jama.2009.1729, indexed in Pubmed: 19934423.

21. Fukuda T, Ohashi-Fukuda N, Matsubara T, et al. Effect of prehospital epinephrine on out-of-hospital cardiac arrest: a report from the national out-of-hospital cardiac arrest data registry in Japan, 2011-2012. Eur J Clin Pharmacol. 2016; 72(10): 1255-1264, doi: 10.1007/s00228-016-2093-2, indexed in Pubmed: 27411936.

22. Goto Y, Maeda T, Goto Y. Effects of prehospital epinephrine during out-of-hospital cardiac arrest with initial non-shockable rhythm: an observational cohort study. Crit Care. 2013; 17(5): R188, doi: 10.1186/cc12872, indexed in Pubmed: 24004456.

23. Neset A, Nordseth T, Kramer-Johansen Jo, et al. Effects of adrenaline on rhythm transitions in out-of-hospital cardiac arrest. Acta Anaesthesiol Scand. 2013; 57(10): 1260-1267, doi: 10.1111/ aas.12184, indexed in Pubmed: 24032427.

24. Machida M, Miura SI, Matsuo K, et al. Effect of intravenous adrenaline before arrival at the hospital in out-of-hospital cardiac arrest. J Cardiol. 2012; 60(6): 503-507, doi: 10.1016/j. jjcc.2012.07.001, indexed in Pubmed: 22890074.

25. Dumas F, Bougouin W, Geri G, et al. Is epinephrine during cardiac arrest associated with worse outcomes in resuscitated patients? J Am Coll Cardiol. 2014; 64(22): 2360-2367, doi: 10.1016/j.jacc.2014.09.036, indexed in Pubmed: 25465423.

26. Fukuda T, Fukuda-Ohashi N, Doi K, et al. Effective pre-hospital care for out-of-hospital cardiac arrest caused by respiratory disease. Heart Lung Circ. 2015; 24(3): 241-249, doi: 10.1016/j. hlc.2014.09.004, indexed in Pubmed: 25445432.

27. Hagihara A, Hasegawa M, Abe T, et al. Prehospital epinephrine use and survival among patients with out-of-hospital cardiac arrest. JAMA. 2012; 307(11): 1161-1168, doi: 10.1001/ jama.2012.294, indexed in Pubmed: 22436956.

28. Hayashi Y, Iwami T, Kitamura T, et al. Impact of early intravenous epinephrine administration on outcomes following outof-hospital cardiac arrest. Circ J. 2012; 76(7): 1639-1645, doi: 10.1253/circj.cj-11-1433, indexed in Pubmed: 22481099. 
29. Herlitz J, Ekström L, Wennerblom B, et al. Adrenaline in outof-hospital ventricular fibrillation. Does it make any difference? Resuscitation. 1995; 29(3): 195-201, doi: 10.1016/03009572(94)00851-6.

30. Herlitz J, Ekström L, Wennerblom B, et al. Predictors of early and late survival after out-of-hospital cardiac arrest in which asystole was the first recorded arrhythmia on scene. Resuscitation. 1994; 28(1): 27-36, doi: 10.1016/0300-9572(94)90051-5.

31. Herlitz J, Ekström L, Wennerblom B, et al. Survival among patients with out-of-hospital cardiac arrest found in electromechanical dissociation. Resuscitation. 1995; 29(2): 97-106, doi: 10.1016/0300-9572(94)00821-v.

32. Holmberg M, Holmberg S, Herlitz J. Low chance of survival among patients requiring adrenaline (epinephrine) or intubation after out-of-hospital cardiac arrest in Sweden. Resuscitation. 2002; 54(1): 37-45, doi: 10.1016/s0300-9572(02)00048-5.

33. Nakahara S, Tomio J, Takahashi H, et al. Evaluation of pre-hospital administration of adrenaline (epinephrine) by emergency medical services for patients with out of hospital cardiac arrest in Japan: controlled propensity matched retrospective cohort study. BMJ. 2013; 347: f6829, doi: 10.1136/bmj.f6829, indexed in Pubmed: 24326886.

34. Robak O, Pruc M, Malysz M, et al. Pre-filled syringes with adrenaline during cardiopulmonary resuscitation in nonshockable rhythms. Pilot randomised crossover simulation study. Disaster Emerg Med J. 2020; 5(2): 79-84, doi: 10.5603/demj.a2020.0025.

35. Blanco J, Madziala M, Szalast P. Which chest compression technique is more fatiguing for rescuer? Disaster Emerg Med J. 2018; 3(4): 150-151, doi: 10.5603/demj.2018.0031.

36. Malysz M, Dabrowski M, Böttiger BW, et al. Resuscitation of the patient with suspected/confirmed COVID-19 when wearing per- sonal protective equipment: A randomized multicenter crossover simulation trial. Cardiol J. 2020; 27(5): 497-506, doi: 10.5603/ CJ.a2020.0068, indexed in Pubmed: 32419128.

37. Kłosiewicz T, Puślecki M, Zalewski R, et al. Assessment of Chest Compression Quality — a systematic review. Disaster Emerg Med J. 2020; 5(1): 49-56, doi: 10.5603/demj.a2020.0010.

38. Majer J, Kaminska H, Wieczorek W, et al. Impact of a CPRrmeter feedback device on chest compression quality performed by nurses - a randomized crossover study. Disaster Emerg Med J. 2018; 3(1): 36-37, doi: 10.5603/demj.2018.0008.

39. Kramer-Johansen Jo, Edelson DP, Losert H, et al. Uniform reporting of measured quality of cardiopulmonary resuscitation (CPR). Resuscitation. 2007; 74(3): 406-417, doi: 10.1016/j.resuscitation.2007.01.024, indexed in Pubmed: 17391831.

40. Sutton RM, Wolfe H, Nishisaki A, et al. Pushing harder, pushing faster, minimizing interruptions... but falling short of 2010 cardiopulmonary resuscitation targets during in-hospital pediatric and adolescent resuscitation. Resuscitation. 2013; 84(12): 1680-1684, doi: 10.1016/j.resuscitation.2013.07.029, indexed in Pubmed: 23954664.

41. Smereka J, Iskrzycki Ł, Makomaska-Szaroszyk E, et al. The effect of chest compression frequency on the quality of resuscitation by lifeguards. A prospective randomized crossover multicenter simulation trial. Cardiol J. 2019; 26(6): 769-776, doi: 10.5603/CJ.a2018.0121, indexed in Pubmed: 30338845.

42. Nolan JP, Deakin CD, Ji C, et al. Intraosseous versus intravenous administration of adrenaline in patients with out-of-hospital cardiac arrest: a secondary analysis of the PARAMEDIC2 placebo-controlled trial. Intensive Care Med. 2020; 46(5): 954-962, doi: 10.1007/s00134-019-05920-7, indexed in Pubmed: 32002593. 